\title{
Intensity-modulated radiotherapy plus nimotuzumab with or without concurrent chemotherapy for patients with locally advanced nasopharyngeal carcinoma
}

This article was published in the following Dove Press journal: OncoTargets and Therapy

\author{
Jianfeng Huangl,* \\ Qinzhou Zou',* \\ Danqi Qian' \\ Leyuan Zhou' \\ Bo Yang' \\ Jianjun $\mathrm{Chu}^{\prime}$ \\ Qingfeng Pang ${ }^{2}$ \\ Kewei Wang ${ }^{2}$ \\ Fuzheng Zhang' \\ 'Department of Radiation Oncology, \\ Affiliated Hospital of Jiangnan \\ University, ${ }^{2}$ Department of \\ Epidemiology, Wuxi Medical School \\ of Jiangnan University, Wuxi, Jiangsu, \\ People's Republic of China \\ *These authors contributed equally \\ to this work
}

Correspondence: Kewei Wang Department of Epidemiology, Wuxi Medical School of Jiangnan University, I800 Lihu Avenue, Wuxi 214 I22,

People's Republic of China

Tel +865I0 85328363

Fax +8651085328605

Emailwkw168@yeah.net

Fuzheng Zhang

Department of Radiation Oncology,

Affiliated Hospital of Jiangnan University,

200 Huihe Road, Wuxi 214062,

People's Republic of China

Tel +865I0 88682111

Fax +865108586032I

Email wxsyzfz@।63.com
Objective: This study aimed to evaluate the safety and efficacy of intensity-modulated radiotherapy (IMRT) plus nimotuzumab with or without concurrent chemotherapy (CCT) for patients with locally advanced nasopharyngeal carcinoma (LA-NPC).

Patients and methods: A total of 50 newly diagnosed patients with LA-NPC treated at the Affiliated Hospital of Jiangnan University between November 2011 and January 2017 were retrospectively analyzed. All patients received the combined treatment modality of nimotuzumab plus IMRT. Nimotuzumab was administered concurrently with IMRT at a weekly dose of $200 \mathrm{mg}$. Neoadjuvant, concurrent or adjuvant chemotherapy with the doublet regimen of taxanes (docetaxel or paclitaxel) plus platinum (cisplatin or nedaplatin) were administered. Among the 50 patients, $43(86.0 \%)$ received $\geq 6$ cycles of nimotuzumab (median 7 cycles, range $2-14$ cycles) and 29 (58.0\%) received two cycles of CCT with docetaxel plus nedaplatin.

Results: With a median follow-up of 28.0 months, the 2-year progression-free survival (PFS) and overall survival were $83.29 \%$ (95\% confidence interval [CI]: 67.93\%-91.72\%) and 97.67\% (95\% CI: 84.62\%-99.67\%), respectively. Both univariate and multivariate analyses revealed that cycles of nimotuzumab were significantly associated with PFS. Patients who received $\geq 6$ cycles of nimotuzumab showed a better PFS than those receiving $<6$ cycles $(P=0.006)$, whereas the addition of CCT failed to improve PFS. Oral mucositis was the most common adverse event, which was recorded as grade 3-4 in $18(36.0 \%)$ patients. Besides, two $(4.0 \%)$ patients experienced nimotuzumab-related anaphylaxis, and no skin rash was found in any patient. Subgroup analysis revealed that the patients who received CCT had more grade 3-4 adverse events as compared to those who did not receive CCT (62.1\% vs $33.3 \%, P=0.045)$.

Conclusion: The regime of nimotuzumab plus IMRT for the treatment of LA-NPC was well tolerated, with encouraging survival data, and it could be an effective treatment alternative for patients with LA-NPC. Further clinical trials are needed to confirm these findings.

Keywords: nasopharyngeal carcinoma, intensity-modulated radiotherapy, nimotuzumab, concurrent chemoradiotherapy, treatment outcome

\section{Introduction}

Nasopharyngeal carcinoma (NPC) is rather common in Southern China and more than $50 \%$ of patients are presented with locally advanced disease (LA-NPC). The optimal treatment of LA-NPC involves a multidisciplinary management. While radiotherapy is the primary treatment modality, the adoption of concurrent chemotherapy (CCT) during the course of radiotherapy remains the standard of care. ${ }^{1}$ However, survival 
in patients with LA-NPC is still only $50 \%-70 \%$ at 5 years, with $30 \%$ of patients experiencing distant metastasis and $10 \%-20 \%$ developing locoregional relapse. ${ }^{2-4}$ Thus, newer treatment modalities, especially the use of molecular targeted agents, concurrently with radiotherapy or with chemoradiotherapy are explored.

The epidermal growth factor receptor (EGFR), a frequently overexpressed tyrosine kinase receptor, plays an important role in carcinogenesis and progression of NPC, and it is associated with poor survival outcome. ${ }^{5,6}$ Great efforts to target the EGFR as a treatment strategy have been made over the last decades. It has been reported that administration of cetuximab, the most commonly used monoclonal antibody against EGFR, concurrently with radiotherapy in locally advanced head and neck squamous cell carcinoma (HNSCC) could significantly improve overall survival (OS) as compared to radiotherapy alone (49.0 months vs 29.3 months, $P=0.03)$. $^{7,8}$ In NPC, the schedule of cetuximab plus radiotherapy with or without chemotherapy has also demonstrated promising preliminary results. ${ }^{9-12}$ However, unexpected increased rates of mucositis, radiation dermatitis and cetuximab-related rash were observed in these studies, which greatly limited its clinical application. Nimotuzumab is a humanized EGFR-blocking antibody with significantly lower rates of mucosal and skin toxicities. ${ }^{13-15}$ In a Phase IIb study reported by Reddy et al, the regimen of nimotuzumab plus radiotherapy or chemoradiation showed significant OS benefit in locally advanced HNSCC. ${ }^{16}$ In NPC, it was reported that the combined therapy of nimotuzumab plus radiotherapy showed promising outcomes in terms of locoregional control and survival. ${ }^{17-19}$ However, the role of adding CCT to nimotuzumab plus radiotherapy remains to be defined.

In this work, 50 newly diagnosed patients with LA-NPC who received the regime of nimotuzumab plus intensitymodulated radiotherapy (IMRT) with or without CCT were retrospectively reviewed. The purpose of this study was to evaluate the safety and efficacy of this treatment modality.

\section{Patients and methods}

\section{Patient population}

Inclusion criteria were as follows: 1) newly diagnosed with histologically confirmed NPC, clinically staged T3-4 or any node-positive disease; 2) no evidence of metastatic disease; 3) no previous treatment with radiotherapy, chemotherapy or EGFR inhibitors; 4) no pregnancy or lactation; 5) no concomitant malignant disease; 6) Eastern Cooperative Oncology Group (ECOG) performance status 2 or lower; and 7) receiving the combinational treatment modality of nimotuzumab plus chemoradiotherapy at initial diagnosis. The study protocol was approved by the institutional Ethics Committee of the Affiliated Hospital of Jiangnan University. Written informed consent was obtained from all patients or their legal guardians before radiotherapy, chemotherapy or nimotuzumab therapy.

Between November 2011 and January 2017, in the Department of Radiation Oncology, Affiliated Hospital of Jiangnan University, 50 patients (median 53 years, range 17-74 years) who met all of the inclusion criteria were enrolled and retrospectively analyzed. Baseline characteristics are listed in Table 1. All patients were staged according to 2010 Union for International Cancer Control (UICC) staging system. Patient workup consisted of medical history, physical examination, hematologic and biochemistry profiles, nasopharyngoscopy, magnetic resonance imaging (MRI) scan of the nasopharynx and neck, chest computed tomography, bone scintigraphy and abdominal ultrasonography.

\section{Treatment methods}

\section{Radiotherapy}

All patients were treated with radical IMRT; the total dose was 70-76 Gy to the planning target volume of the primary tumor and involved cervical lymph nodes, 60 Gy to the subclinical disease around the primary tumor and upper neck and 54 Gy to the lower neck and supraclavicular region.

Table I Baseline characteristics of patients

\begin{tabular}{ll}
\hline Characteristic & $\begin{array}{l}\text { Number } \\
\text { of patients }\end{array}$ \\
\hline Total & 50 \\
Gender & \\
Male & 40 \\
Female & 10 \\
Age, years & \\
Median & 53 \\
Range & $17-74$ \\
>50 & 20 \\
S50 & 30 \\
Clinical T category & \\
TI & 3 \\
T2 & 20 \\
T3 & 19 \\
T4 & 8 \\
Clinical N category & \\
N0 & 3 \\
NI & 11 \\
N2 & 27 \\
N3 & 9 \\
ICC stage & \\
III & \\
IVa & \\
IVb & 26 \\
\hline
\end{tabular}

Abbreviations: T, tumor; N, lymph node; UICC, Union for International Cancer Control. 
Radiotherapy was delivered once daily, five fractions per week. Dose constraints to adjacent critical organs were applied according to the Radiation Therapy Oncology Group (RTOG) 0225 protocol.

\section{Chemotherapy and nimotuzumab therapy}

The treatment modalities of chemotherapy and nimotuzumab are listed in Table 2. Nimotuzumab was administered concurrently with IMRT at a weekly dose of $200 \mathrm{mg}$ in all patients. Among them, $43(86.0 \%)$ received $\geq 6$ cycles of nimotuzumab (median 7 cycles, range $2-14$ cycles). Neoadjuvant, concurrent or adjuvant chemotherapy with the doublet regimen of taxanes (docetaxel or paclitaxel) plus platinum (cisplatin or nedaplatin) were administered. The general course was 4-6 cycles, with two cycles of neoadjuvant and CCT, and 1-2 cycles of adjuvant chemotherapy. While two patients received neoadjuvant chemotherapy alone, 19 patients received neoadjuvant plus adjuvant chemotherapy, and the other 29 patients received neoadjuvant, concurrent plus adjuvant chemotherapy. Chemotherapy regimens: majority of patients $(90.0 \%)$ received the regime of docetaxel (75 $\mathrm{mg} / \mathrm{m}^{2}$ on day 1) plus nedaplatin $\left(80 \mathrm{mg} / \mathrm{m}^{2}\right.$ on day 2$)$, paclitaxel $\left(135 \mathrm{mg} / \mathrm{m}^{2}\right.$ on day 1$)$ or cisplatin $\left(25 \mathrm{mg} / \mathrm{m}^{2}\right.$ on days 1-3) was administered in four and one patients, respectively: four received paclitaxel plus nedaplatin and the other one received docetaxel plus cisplatin. Among the 29 patients who received CCT, two cycles of docetaxel plus nedaplatin were administered.

\section{Patient evaluation and follow-up}

Nimotuzumab and chemotherapy toxicities were graded by the National Cancer Institute Common Toxicity Criteria (NCI CTCAE, version 3.0; https://ctep.cancer.gov/protocolDevelopment/electronic applications/docs/ctcaev3.pdf), whereas radiotherapy-related toxicities were assessed using

Table 2 The treatment modalities of chemotherapy and nimotuzumab combined with IMRT in all patients

\begin{tabular}{ll}
\hline The treatment modalities & $\begin{array}{l}\text { Number } \\
\text { of patients }\end{array}$ \\
\hline Chemotherapy & 2 \\
NAC & 19 \\
NAC + AC & 29 \\
NAC + CCT + AC & \\
Nimotuzumab therapy, cycles & 7 \\
Median & $2-14$ \\
Range & 43 \\
$\geq 6$ & 7 \\
$<6$ & 7
\end{tabular}

Abbreviations: IMRT, intensity-modulated radiotherapy; NAC, neoadjuvant chemotherapy; AC, adjuvant chemotherapy; CCT, concurrent chemotherapy. the Acute and Late Radiation Morbidity Scoring Criteria of RTOG (CTCAE 3.0, https://www.rtog.org/ResearchAssociates/AdverseEventReporting.aspx). The evaluation of disease response was according to the Response Evaluation Criteria in Solid Tumors (RECIST, version 1.1; https://ctep.cancer.gov/ protocolDevelopment/docs/recist guideline.pdf). Treatment response and toxicities were assessed and recorded weekly during radiotherapy. All patients were followed at intervals of 3 months after the completion of radiotherapy for 3 years, biannually for the next 2 years and annually thereafter.

\section{Statistical analysis}

The STAT software, version 12.0, was used for statistical analysis. The main purpose of this work was to evaluate the efficacy and safety of nimotuzumab plus chemoradiation in patients with LA-NPC. The estimated OS and progressionfree survival (PFS) were calculated by the Kaplan-Meier method. Univariate analysis was performed with log-rank test. Impact of prognostic factors on PFS was evaluated in a multifactor Cox model. OS was measured from the time of pathological diagnosis to the date of death due to any cause or the date of last follow-up. PFS was determined as the time between the date of pathological diagnosis and the first occurrence of locoregional or distant recurrence or the date of last follow-up.

\section{Results \\ Treatment outcomes}

Objective response rate (ORR) was $100 \%$ at 3 months after the completion of IMRT, with $44(88.0 \%)$ complete responses and $6(12.0 \%)$ partial responses. With a median follow-up of 28.0 months (range, 6.4-69.1 months), the 2-year PFS and OS were $83.29 \%$ (95\% confidence interval [CI]: 67.93\%-91.72\%) and 97.67\% (95\% CI: 84.62\%-99.67\%) (Figures 1 and 2).

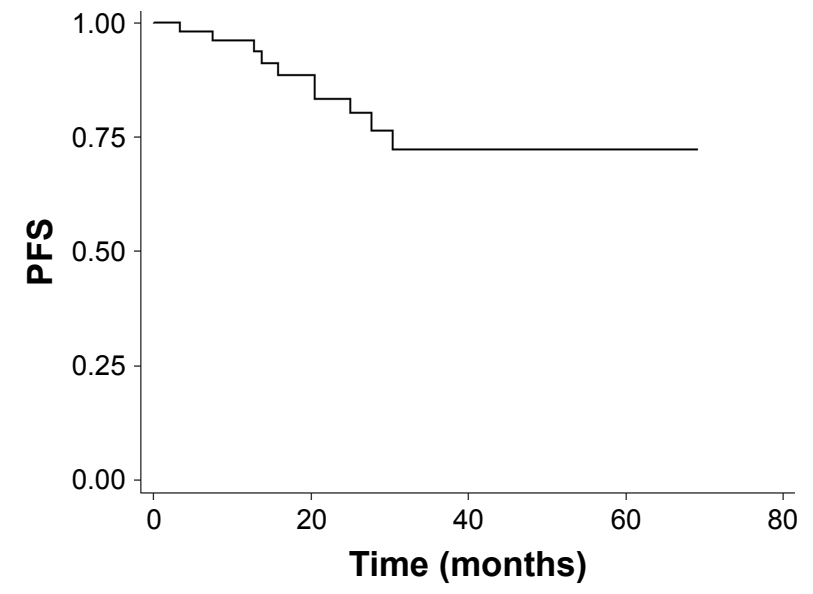

Figure I Kaplan-Meier survival curve of PFS (enrolled population). Abbreviation: PFS, progression-free survival. 


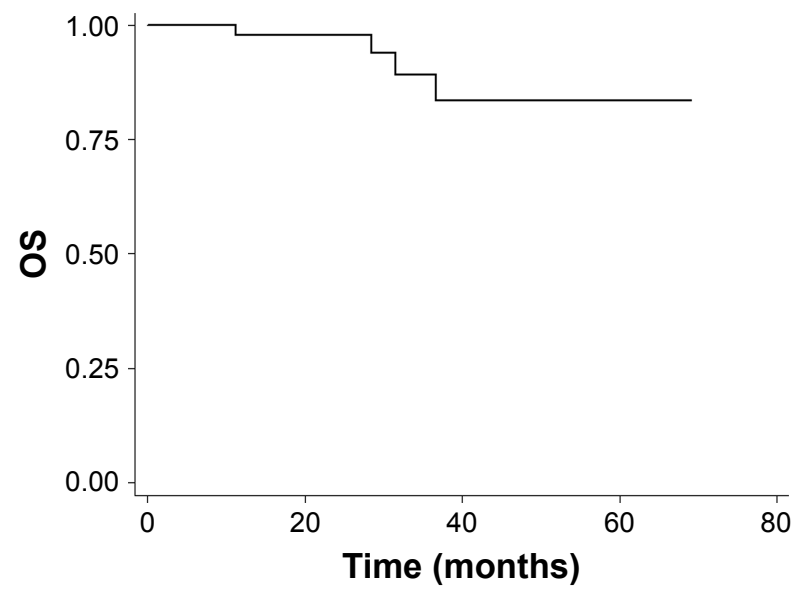

Figure 2 Kaplan-Meier survival curve of OS (enrolled population). Abbreviation: OS, overall survival.

At the last follow-up visit, a total of 10 patients failed with distant metastasis: six with bone metastases only, two with liver metastases only, one with liver and bone metastases, one with lung and bone involvement, and four of them had died of disease progression. Locoregional recurrence was not observed in our cohort.

\section{Prognostic factors}

Subgroup analysis was performed to examine the potential prognostic factors for predicting PFS, including gender, age, clinical $\mathrm{T}$ category, clinical $\mathrm{N}$ category,
UICC stage, CCT and cycles of nimotuzumab (Table 3 ). Both univariate and multivariate analyses showed that cycles of nimotuzumab were significantly associated with PFS. Patients who received $\geq 6$ cycles of nimotuzumab $(\mathrm{n}=43)$ showed a better PFS than those receiving $<6$ cycles $(n=7)(P=0.006$, Figure 3$)$. No significant difference was observed among other groups of patients with different gender, age, clinical $\mathrm{T}$ category, clinical $\mathrm{N}$ category, UICC stage, as well as those receiving CCT or not $(P>0.05)$, although UICC stage (stage II-III vs stage IV) appears to be a marginal prognostic factor for PFS in multivariate analysis $(P=0.058$, Figure 4$)$.

\section{Treatment toxicities}

The treatment-related acute toxicities during IMRT plus nimotuzumab are listed in Table 4. Altogether, the oral mucositis was the most common adverse event, which was recorded as grade 3 in $16(32.0 \%)$ patients and grade 4 in two $(4.0 \%)$ patients. In addition, nine $(18.0 \%)$ patients suffered with grade 3 neutropenia, three $(6.0 \%)$ with grade 3 dermatitis, and one (2.0\%) with grade 3 anemia. It should be noted that two $(4.0 \%)$ patients had experienced nimotuzumabrelated anaphylaxis, while no acne-like rash was found in any patient.

Table 5 summarizes grade 3-4 acute adverse events during IMRT plus nimotuzumab. All patients were

Table 3 Impact of prognostic factors on PFS by univariate and multivariate analysis

\begin{tabular}{|c|c|c|c|c|c|}
\hline \multirow[t]{2}{*}{ Variable } & \multirow[t]{2}{*}{ No } & \multicolumn{2}{|c|}{ Univariate analysis } & \multicolumn{2}{|c|}{ Multivariate analysis } \\
\hline & & $P$-value & HR (95\% Cl) & $P$-value & HR $(95 \% \mathrm{Cl})$ \\
\hline Gender & & 0.268 & & 0.167 & \\
\hline Male & 40 & & $3.224(0.407-25.571)$ & & $4.939(0.512-47.644)$ \\
\hline Female & 10 & & & & \\
\hline Age, years & & 0.859 & & $0.48 I$ & \\
\hline$>50$ & 30 & & $0.892(0.25 I-3.166)$ & & $0.583(0.130-2.615)$ \\
\hline$\leq 50$ & 20 & & & & \\
\hline Clinical T category & & 0.310 & & 0.608 & \\
\hline TI-2 & 23 & & $2.017(0.52 I-7.805)$ & & $0.619(0.099-3.880)$ \\
\hline T3-4 & 27 & & & & \\
\hline Clinical $\mathrm{N}$ category & & 0.302 & & 0.125 & \\
\hline No-2 & $4 I$ & & $2.041(0.526-7.919)$ & & $0.139(0.011-1.733)$ \\
\hline N3 & 9 & & & & \\
\hline UICC stage & & 0.114 & & 0.058 & \\
\hline II-III & 34 & & $2.726(0.785-9.465)$ & & II.206 (0.92I-136.3 I5) \\
\hline IV & 16 & & & & \\
\hline Concurrent chemotherapy & & 0.302 & & 0.422 & \\
\hline Yes & 29 & & $2.042(0.526-7.919)$ & & $2.096(0.344-12.779)$ \\
\hline No & 21 & & & & \\
\hline Cycles of nimotuzumab & & 0.010 & & 0.006 & \\
\hline$<6$ & 7 & & $0.184(0.05 I-0.669)$ & & $0.076(0.012-0.47 I)$ \\
\hline$\geq 6$ & 43 & & & & \\
\hline
\end{tabular}

Abbreviations: PFS, progression-free survival; T, tumor; N, lymph node; UICC, Union for International Cancer Control; HR, hazard ratio; Cl, confidence interval. 


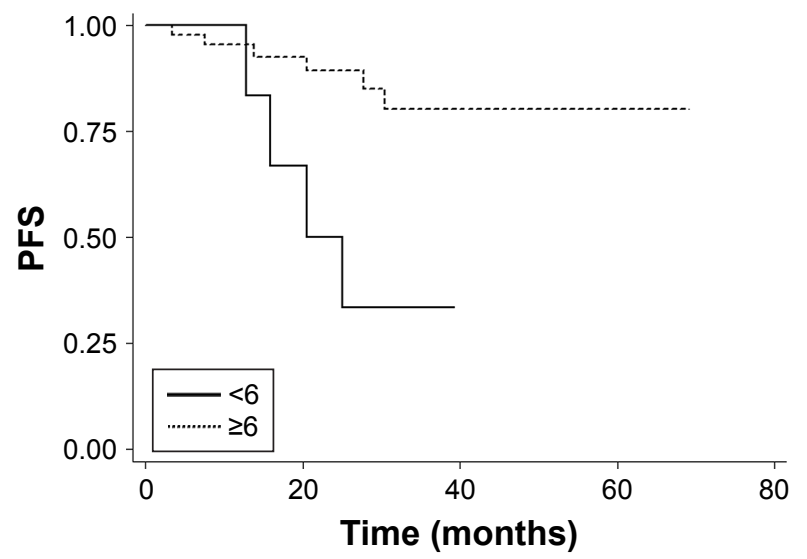

Figure 3 Kaplan-Meier survival curves of PFS for patients receiving $\geq 6$ versus $<6$ cycles of nimotuzumab.

Abbreviation: PFS, progression-free survival.

divided into two groups according to whether CCT was administered or not. Adverse events of grade 3-4 were observed in $18(62.1 \%)$ patients for the CCT group $(n=29)$ and in seven $(33.3 \%$ ) patients for those who did not receive $\operatorname{CCT}(n=21)$; the difference between the two groups was statistically significant $(P=0.045)$. We also found that the incorporation of CCT in our cohort showed a trend of more serious oral mucositis and neutropenia. For patients who received CCT, grade 3-4 acute oral mucositis and neutropenia occurred in $12(41.4 \%)$ and seven $(24.1 \%)$ patients, respectively, while it was recorded as six (28.6\%) and two $(9.5 \%)$, respectively, for those patients who did not receive CCT $(P>0.05)$.

Xerostomia was the most common late toxicity and the degree was alleviated over time during follow-up. Three patients experienced mild-to-moderate cellulitis in maxillofacial region and neck and recovered after pharmacotherapy. One patient suffered from nasopharyngeal bleeding, stopping after symptomatic treatment. At the last follow-up,

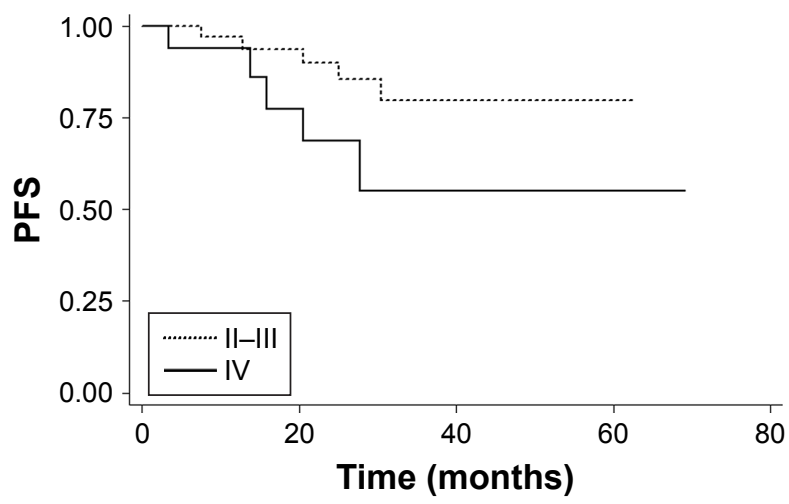

Figure 4 Kaplan-Meier survival curves of PFS for patients with stage II-III versus stage IV.

Abbreviation: PFS, progression-free survival.
Table 4 The frequency of acute toxicities during IMRT plus nimotuzumab

\begin{tabular}{llll}
\hline Acute toxicities & \multicolumn{3}{l}{ Number of patients (\%) } \\
\cline { 2 - 4 } & Grade I-2 & Grade 3 & Grade 4 \\
\hline Neutropenia & $23(46.0)$ & $9(18.0)$ & $0(0)$ \\
Thrombocytopenia & $4(8.0)$ & $0(0)$ & $0(0)$ \\
Anemia & $5(10.0)$ & $\mathrm{I}(2.0)$ & $0(0)$ \\
Oral mucositis & $32(64.0)$ & $16(32.0)$ & $2(4.0)$ \\
Dermatitis & $44(88.0)$ & $3(6.0)$ & $0(0)$ \\
Nimotuzumab-related rash & $0(0)$ & $0(0)$ & $0(0)$ \\
Nimotuzumab-related anaphylaxis & $\mathrm{I}(2.0)$ & $\mathrm{I}(2.0)$ & $0(0)$ \\
\hline Abbreviation: IMRT, intensity-modulated radiotherapy. & &
\end{tabular}

radiation-induced temporal lobe necrosis, cranial nerve palsy, myelopathy, osteonecrosis and pituitary damage were not observed. There were no treatment-related deaths.

\section{Discussion}

In the study presented here, the combinational treatment modality of nimotuzumab plus chemoradiation was retrospectively evaluated in stage T3-4 or any node-positive NPC patients. The ORR at 3 months after the completion of IMRT was $100 \%$, with a 2 -year PFS of $83.29 \%$ and a 2 -year OS of $97.67 \%$. The preliminary outcome data appear encouraging as compared to historical data of concurrent chemoradiation, which remains the mainstream for the management of LA-NPC patients..$^{20,21}$

The updated study by collaborative group of MetaAnalysis of Chemotherapy in Nasopharyngeal Carcinoma has revealed that, compared with radiotherapy alone, the addition of CCT could significantly improve OS in patients with LA-NPC. ${ }^{22}$ However, with the optimized local control by IMRT and the use of targeted agents, the role of CCT was under increasing doubts and challenges. A retrospective analysis conducted by Sun et al revealed that CCT failed to improve survival in the IMRT setting. ${ }^{3}$ Most recently, You et al published findings of their matched case-control study of cetuximab or nimotuzumab plus IMRT versus cisplatin plus IMRT for stage II-IVb NPC, there was no significant difference between the two groups in terms of disease-free survival (3-year, $86.7 \%$ vs $86.2 \%, P>0.05)$ and $\mathrm{OS}(91.7 \%$ vs $91.9 \%, P>0.05) .{ }^{19}$ Also the benefit of CCT is still uncertain for patients treated with IMRT plus EGFR inhibitors. In the study reported by Niu et al, 33 patients with LA-NPC were given the regime IMRT plus cetuximab with or without CCT; at a median follow-up of 40.0 months, the 3-year PFS were $83.3 \%$ and $61.0 \%$, respectively, with no statistical significance $(P=0.574) .{ }^{11}$ In our cohort, among the 50 patients treated with IMRT plus nimotuzumab, 29 (58.0\%) received two cycles of CCT with docetaxel plus nedaplatin, and no 
Table 5 The frequency of grade 3-4 acute adverse events during IMRT plus nimotuzumab with or without CCT

\begin{tabular}{|c|c|c|c|c|c|c|}
\hline \multirow[t]{2}{*}{ CCT } & \multirow[t]{2}{*}{ No } & \multicolumn{5}{|c|}{ Grade $3-4$ adverse events (\%) } \\
\hline & & Total & Mucositis & Neutropenia & Anemia & Dermatitis \\
\hline With & 29 & $18(62.1)$ & $12(4 \mid .4)$ & $7(24.1)$ & I (3.4) & $2(6.9)$ \\
\hline Without & 21 & $7(33.3)$ & $6(28.6)$ & $2(9.5)$ & $0(0)$ & $\mathrm{I}(4.8)$ \\
\hline$P$-value & N/A & 0.045 & 0.352 & 0.340 & 1.000 & 0.756 \\
\hline
\end{tabular}

Abbreviations: IMRT, intensity-modulated radiotherapy; CCT, concurrent chemotherapy; N/A, not available.

benefit of PFS was observed in comparison with those not receiving $\operatorname{CCT}(P=0.422)$. These results strongly suggest that nimotuzumab combined with IMRT is an effective alternative to the standard care of concurrent chemoradiation for patients with LA-NPC, especially for those who cannot tolerate CCT.

Interestingly, our study also demonstrated that patients who received $\geq 6$ cycles of nimotuzumab showed a statistically significant improvement in PFS compared with those who received $<6$ cycles $(P=0.006)$. Although the dose-effect relationship of nimotuzumab on OS is still to be determined, our preliminary results indicated that sufficient dosage of nimotuzumab is very important when used along with IMRT. Similar findings have showed up in other studies of EGFR inhibitors. In a retrospective analysis conducted by Wang et al, the regime of high-dose nimotuzumab $(>1,200 \mathrm{mg})$ plus radiotherapy was associated with improved 5-year OS as compared to the low-dose group for patients with esophageal carcinoma. ${ }^{23}$ In LA-NPC, it has also been reported that more cycles of cetuximab $(\geq 7)$ plus IMRT showed a better 3 -year PFS than less cycles $(79.1 \%$ vs $31.2 \%, P=0.050) .{ }^{11}$ However, these results should be regarded as preliminary because of the relatively small sample size and short follow-up, as well as the bias in patient selection. Further studies to determine the best and appropriate drug dose are necessary.

It is well known that addition of cetuximab to IMRT could result in unexpectedly increased toxic effects. ${ }^{11,12,24-26}$ In these studies, the skin rash (all grades) occurred in $85.8 \%-100 \%$ of patients, and the frequency of grade 3-4 skin rash, mucositis and dermatitis was reported as $10 \%-39.4 \%, 66.7 \%-87 \%$ and $18.2 \%-25 \%$, respectively. To our excitement, in the study presented here, no skin rash was observed in any patient, and the grade 3-4 mucositis and dermatitis were recorded as $36.0 \%$ and $6.0 \%$, respectively, which were much lower than the values reported in the cetuximab-based studies, and a similar survival of 2-year PFS was found in our cohort as compared to the cetuximab setting. ${ }^{12}$ The favorable normal tissue toxicity results can be attributed to the unique safety profile of nimotuzumab, which requires bivalent binding to tumor cells expressing moderate-to-high EGFR, thus sparing normal tissues where EGFR density is low. ${ }^{27}$
As with other studies of IMRT for LA-NPC, the oral mucositis was the most common acute toxicity. In our study, subgroup analysis of treatment toxicities during IMRT plus nimotuzumab revealed that the patients who received CCT had more grade 3-4 adverse events as compared to those who did not receive CCT $(62.1 \%$ vs $33.3 \%, P=0.045)$, with grade $3-4$ mucositis of $41.4 \%$ versus $28.6 \%(P=0.352)$, grade 3-4 neutropenia of $24.1 \%$ versus $9.5 \%(P=0.340)$ and grade 3-4 dermatitis of $6.9 \%$ versus $4.8 \%(P=0.756)$. These results, together with the survival data as mentioned earlier, further call into question the benefit of adding CCT to IMRT plus nimotuzumab for the treatment of LA-NPC.

\section{Conclusion}

Taken together, our study showed that the combined treatment modality of nimotuzumab plus IMRT for LA-NPC was well tolerated, with encouraging survival data, and it could be an effective alternative treatment for patients with LANPC. However, further clinical trials are needed to confirm these findings.

\section{Acknowledgments}

This work was supported by grants from program of Wuxi Young Medical Talents (No QNRC059) and Wuxi Medical Development Discipline (No FZXK004).

\section{Disclosure}

The authors report no conflicts of interest in this work.

\section{References}

1. Chua MLK, Wee JTS, Hui EP, Chan ATC. Nasopharyngeal carcinoma. Lancet. 2016;387(10022):1012-1024.

2. Ng WT, Lee MC, Hung WM, et al. Clinical outcomes and patterns of failure after intensity-modulated radiotherapy for nasopharyngeal carcinoma. Int J Radiat Oncol Biol Phys. 2011;79(2):420-428.

3. Sun X, Su S, Chen C, et al. Long-term outcomes of intensity-modulated radiotherapy for 868 patients with nasopharyngeal carcinoma: an analysis of survival and treatment toxicities. Radiother Oncol. 2014; 110(3):398-403.

4. Ou X, Zhou X, Shi Q, et al. Treatment outcomes and late toxicities of 869 patients with nasopharyngeal carcinoma treated with definitive intensity modulated radiation therapy: new insight into the value of total dose of cisplatin and radiation boost. Oncotarget. 2015;6(35):38381-38397.

5. Ooft ML, Braunius WW, Heus P, et al. Prognostic significance of the EGFR pathway in nasopharyngeal carcinoma: a systematic review and meta-analysis. Biomark Med. 2015;9(10):997-1010. 
6. Zhang P, Wu SK, Wang Y, et al. p53, MDM2, eIF4E and EGFR expression in nasopharyngeal carcinoma and their correlation with clinicopathological characteristics and prognosis: a retrospective study. Oncol Lett. 2015;9(1):113-118.

7. Bonner JA, Harari PM, Giralt J, et al. Radiotherapy plus cetuximab for locoregionally advanced head and neck cancer: 5-year survival data from a phase 3 randomised trial, and relation between cetuximabinduced rash and survival. Lancet Oncol. 2010;11(1):21-28.

8. Bonner JA, Harari PM, Giralt J, et al. Radiotherapy plus cetuximab for squamous-cell carcinoma of the head and neck. $N$ Engl J Med. 2006; 354(6):567-578.

9. Xia WX, Liang H, Lv X, et al. Combining cetuximab with chemoradiotherapy in patients with locally advanced nasopharyngeal carcinoma: a propensity score analysis. Oral Oncol. 2017;67:167-174.

10. Wu X, Huang J, Liu L, et al. Cetuximab concurrent with IMRT versus cisplatin concurrent with IMRT in locally advanced nasopharyngeal carcinoma: a retrospective matched case-control study. Medicine (Baltimore). 2016;95(39):e4926.

11. Niu X, Hu C, Kong L. Experience with combination of cetuximab plus intensity-modulated radiotherapy with or without chemotherapy for locoregionally advanced nasopharyngeal carcinoma. J Cancer Res Clin Oncol. 2013;139(6):1063-1071.

12. Ma BB, Kam MK, Leung SF, et al. A phase II study of concurrent cetuximab-cisplatin and intensity-modulated radiotherapy in locoregionally advanced nasopharyngeal carcinoma. Ann Oncol. 2012;23(5): 1287-1292.

13. Crombet T, Osorio M, Cruz T, et al. Use of the humanized anti-epidermal growth factor receptor monoclonal antibody h-R3 in combination with radiotherapy in the treatment of locally advanced head and neck cancer patients. J Clin Oncol. 2004;22(9):1646-1654.

14. Huang JF, Zhang FZ, Zou QZ, et al. Induction chemotherapy followed by concurrent chemoradiation and nimotuzumab for locoregionally advanced nasopharyngeal carcinoma: preliminary results from a phase II clinical trial. Oncotarget. 2017;8(2):2457-2465.

15. Westphal M, Heese O, Steinbach JP, et al. A randomised, open label phase III trial with nimotuzumab, an anti-epidermal growth factor receptor monoclonal antibody in the treatment of newly diagnosed adult glioblastoma. Eur J Cancer. 2015;51(4):522-532.

16. Reddy BK, Lokesh V, Vidyasagar MS, et al. Nimotuzumab provides survival benefit to patients with inoperable advanced squamous cell carcinoma of the head and neck: a randomized, open-label, phase IIb, 5-year study in Indian patients. Oral Oncol. 2014;50(5):498-505.
17. Huang XD, Yi JL, Gao L, et al. Multi-center phase II clinical trial of humanized anti-epidermal factor receptor monoclonal antibody h-R3 combined with radiotherapy for locoregionally advanced nasopharyngeal carcinoma. Zhonghua Zhong Liu Za Zhi. 2007;29(3): 197-201.

18. Zhai RP, Ying HM, Kong FF, et al. Experience with combination of nimotuzumab and intensity-modulated radiotherapy in patients with locoregionally advanced nasopharyngeal carcinoma. Onco Targets Ther. 2015;8:3383-3390.

19. You R, Sun R, Hua YJ, et al. Cetuximab or nimotuzumab plus intensitymodulated radiotherapy versus cisplatin plus intensity-modulated radiotherapy for stage II-IVb nasopharyngeal carcinoma. Int J Cancer. 2017;141(6):1265-1276.

20. Chan AT, Teo PM, Ngan RK, et al. Concurrent chemotherapyradiotherapy compared with radiotherapy alone in locoregionally advanced nasopharyngeal carcinoma: progression-free survival analysis of a phase III randomized trial. J Clin Oncol. 2002;20(8): 2038-2044.

21. Lee AW, Lau WH, Tung SY, et al. Preliminary results of a randomized study on therapeutic gain by concurrent chemotherapy for regionallyadvanced nasopharyngeal carcinoma: NPC-9901 Trial by the Hong Kong Nasopharyngeal Cancer Study Group. J Clin Oncol. 2005;23(28): 6966-6975.

22. Blanchard $\mathrm{P}$, Lee A, Marguet S, et al. Chemotherapy and radiotherapy in nasopharyngeal carcinoma: an update of the MAC-NPC metaanalysis. Lancet Oncol. 2015;16(6):645-655.

23. Wang C, Fu X, Cai X, et al. High-dose nimotuzumab improves the survival rate of esophageal cancer patients who underwent radiotherapy. Onco Targets Ther. 2016;9:117-122.

24. He X, Xu J, Guo W, Jiang X, Wang X, Zong D. Cetuximab in combination with chemoradiation after induction chemotherapy of locoregionally advanced nasopharyngeal carcinoma: preliminary results. Future Oncol. 2013;9(10):1459-1467.

25. Feng HX, Guo SP, Li GR, et al. Toxicity of concurrent chemoradiotherapy with cetuximab for locoregionally advanced nasopharyngeal carcinoma. Med Oncol. 2014;31(9):170.

26. Li Y, Chen QY, Tang LQ, et al. Concurrent chemoradiotherapy with or without cetuximab for stage II to IVb nasopharyngeal carcinoma: a case-control study. BMC Cancer. 2017;17(1):567.

27. Ramakrishnan MS, Eswaraiah A, Crombet T, et al. Nimotuzumab, a promising therapeutic monoclonal for treatment of tumors of epithelial origin. MAbs. 2009;1(1):41-48.
OncoTargets and Therapy

\section{Publish your work in this journal}

OncoTargets and Therapy is an international, peer-reviewed, open access journal focusing on the pathological basis of all cancers, potential targets for therapy and treatment protocols employed to improve the management of cancer patients. The journal also focuses on the impact of management programs and new therapeutic agents and protocols on

\section{Dovepress}

patient perspectives such as quality of life, adherence and satisfaction. The manuscript management system is completely online and includes a very quick and fair peer-review system, which is all easy to use. Visit http://www.dovepress.com/testimonials.php to read real quotes from published authors. 